Cahiers de géographie du Québec

\title{
Attention médiatique et politique du développement durable à
} Montréal, 1987-2009

\section{Media attention and sustainable development policy in}

Montréal, 1987-2009

\section{Atención periodística y política del desarrollo sostenido en Montreal, 1987-2009}

\section{Edouard-Alexis Sigward et Catherine Trudelle}

Volume 60, numéro 169, avril 2016

Version originale soumise en août 2015. Version révisée reçue en février 2016.

URI : https://id.erudit.org/iderudit/1038667ar

DOI : https://doi.org/10.7202/1038667ar

Aller au sommaire du numéro

Éditeur(s)

Département de géographie de l’Université Laval

ISSN

0007-9766 (imprimé)

1708-8968 (numérique)

Découvrir la revue

Citer cet article

Sigward, E.-A. \& Trudelle, C. (2016). Attention médiatique et politique du développement durable à Montréal, 1987-2009. Cahiers de géographie du Québec, 60(169), 129-156. https://doi.org/10.7202/1038667ar
Résumé de l'article

Pour devenir un véritable projet de société, le développement durable (DD) doit s'appuyer sur l'effort collectif de tous les acteurs de la société impliqués dans le développement socioterritorial. Il est donc essentiel que ceux-ci maîtrisent l'ensemble des contours du concept. Contribuant à la formation de l'opinion des citoyen(ne)s et des décideurs, les médias de masse jouent un rôle particulier à cet égard. De fait, de la manière dont les sphères médiatique et politique interprètent, s'approprient et portent le discours du DD dépendent la qualité et la viabilité de son implantation. A travers les approches quantitative et qualitative des cycles d'attention médiatique et de l'analyse comparative de contenus, cet article étudie la résonnance médiatique et politique du DD à Montréal entre 1987 et 2009. Au terme de la recherche, les résultats démontrent la possibilité d'un effet structurant du Premier plan stratégique de développement durable de la collectivité montréalaise (2005-2009) sur les cycles d'attention médiatique du DD. Ils révèlent aussi que le Plan et la presse écrite montréalaise n'insistent pas sur les mêmes dimensions du DD, même si leurs interprétations respectives ont tendance à converger davantage à partir des années 2000 


\section{Attention médiatique et politique du développement durable à Montréal, 1987-2009}

\author{
Media attention and sustainable \\ development policy in Montréal, 1987-2009 \\ Atención periodística y política del desarrollo \\ sostenido en Montreal, 1987-2009
}

\author{
Edouard-Alexis SIGWARD et Catherine TRUDELLE \\ Département de géographie \\ Université du Québec à Montréal \\ Edouard.Sigward@gmail.com \\ Trudelle.Catherine@uqam.ca
}

\section{Résumé}

Pour devenir un véritable projet de société, le développement durable (DD) doit s'appuyer sur l'effort collectif de tous les acteurs de la société impliqués dans le développement socioterritorial. Il est donc essentiel que ceux-ci maîtrisent l'ensemble des contours du concept. Contribuant à la formation de l'opinion des citoyen(ne)s et des décideurs, les médias de masse jouent un rôle particulier à cet égard. De fait, de la manière dont les sphères médiatique et politique interprètent, s'approprient et portent le discours du DD dépendent la qualité et la viabilité de son implantation. À travers les approches quantitative et qualitative des cycles d'attention médiatique et de l'analyse comparative de contenus, cet article étudie la résonnance médiatique et politique du DD à Montréal entre 1987 et 2009. Au terme de la recherche, les résultats démontrent la possibilité d'un effet structurant du Premier plan stratégique de développement durable de la collectivité montréalaise (2005-2009) sur les cycles d'attention médiatique du DD. Ils révèlent aussi que le Plan et la presse écrite montréalaise n'insistent pas sur les mêmes dimensions du $\mathrm{DD}$, même si leurs interprétations respectives ont tendance à converger davantage à partir des années 2000 .

\section{Mots-clés}

Développement durable, acteurs sociaux, presse montréalaise, cycles d'attention médiatique, Premier plan stratégique de développement durable de la collectivité montréalaise (2005-2009).

\section{Abstract}

To become a collective social project, sustainable development (SD) needs the support of all citizens and decision makers. Essential to this process is an understanding of the concept by all those who contribute to social and territorial development. Through their influence in shaping public opinion and political thinking, the media play a major role in this respect. The applicability and staying power of the achievement of SD in a society depend on how the media and political spheres comprehend, integrate and communicate the sustainable development discourse. The goal of this article is to examine the media and political response to SD in Montréal between 1987 and 2009. We analyse the ways in which media coverage of the issue of sustainability and the implementation of Montréal's First Strategic Plan for Sustainable Development (2005-2009) are connected, on both a quantitative and qualitative level. Our results demonstrate possible structural effects of the Strategic Plan on issue-attention cycles around the question of sustainability in the Montréal news media. They also show that the Plan and the print media do not always stress the same areas of $\mathrm{SD}$, although the interpretations they present tend to more closely converge from the early 2000 s onwards.

\section{Keywords}

Sustainable development, sustainability, Montréal print media, issue-attention cycles, Montréal's First Strategic Plan for Sustainable Development (2005-2009). 


\section{Resumen}

Para llegar a ser un verdadero proyecto de sociedad, el desarrollo sostenido (DD o développement durable) debe apoyarse sobre el esfuerzo colectivo de todos los actores comprometidos en el desarrollo socio-territorial. Es esencial que éstos dominen el conjunto de las dimensiones del concepto. La prensa juega un rol particular al respecto, pues contribuye a la formación de la opinión de ciudadanas, ciudadanos y de los que tienen el poder de tomar decisiones. Efectivamente, de la manera cómo las esferas políticas e informativas interpreten, se apropien y asuman el discurso del $D D$ dependerá la calidad y la viabilidad de su instalación. Con un enfoque cuantitativo y cualitativo de los ciclos de atención mediática y del análisis comparativo de contenido, este artículo estudia la resonancia periodística y política del $D D$ en Montreal, entre 1987 y 2009. Los resultados de la investigación demuestran la posibilidad de un efecto estructurante del Primer Plan Estratégico de Desarrollo Sostenido de la colectividad montrealesa (2005-2009), sobre los ciclos de atención mediática del DD. Éstos muestran que el Plan de la prensa escrita de Montreal no insisten sobre las mismas dimensiones del $D D$, aunque sus interpretaciones tiendan a converger mejor a partir de los años 2000.

\section{Palabras claves}

Desarrollo sostenido, actores sociales, prensa de Montreal, ciclos de atención mediática, Primer Plan Estratégico de Desarrollo Sostenido de la colectividad de Montreal.

\section{Introduction}

En l'espace de deux décennies, la rhétorique du développement durable s'est installée dans la plupart des discours animant la société et semble être devenue la nouvelle source d'inspiration de la croissance. Cependant, si aujourd'hui l'expression développement durable ne semble plus échapper à personne, les tenants et aboutissants du concept auxquels elle renvoie sont souvent mal maîtrisés, voire carrément incompris, par les acteurs du développement économique et socioterritorial chargés de sa mise en œuvre. De fait, le développement durable est caractérisé par un flou - tant conceptuel que sémantique - qui ne facilite ni sa compréhension théorique, ni son application pratique. Cette difficulté d'interprétation donne lieu à une mauvaise compréhension des enjeux transversaux sous-tendus par l'idée même du développement durable et renforce la tendance des différents acteurs à ne considérer que les seules dimensions du concept pouvant servir leur périmètre d'activité particulier (Vivien, 2003; Besancenot, 2009).

Véritable projet d'une société en devenir, le développement durable (DD) se trouve à la confluence des sphères économique, environnementale et sociale. Il invite les sociétés contemporaines à appréhender le monde de manière écosystémique et à endosser leur part de responsabilité à l'égard d'un environnement planétaire dont elles conviennent désormais qu'il est en sursis (CMED, 1989; Anton, 2009). Dans la mesure où il intervient sur le plan idéologique et aspire à gagner les esprits de tous au nom de l'abstraction des générations futures, le DD est un concept éminemment universaliste (Moreau Defarges, 2008). Dans cette perspective, il appelle la participation de tous les acteurs traditionnels de la société et la mobilisation des citoyens qui se voient ainsi responsabilisés au sort de la planète entière (Felli, 2008). Toutefois, comme le note Baddache (2006: 40-41), «les individus, les citoyens et les pouvoirs publics n’ont pas «naturellement» la fibre du développement durable». Les différentes interprétations que ces acteurs font du DD engagent donc un questionnement déterminant pour son implantation par, dans et pour la société. 
Aujourd'hui plus que jamais, le monde observe et s'observe à travers le prisme des médias de masse. La société est sans cesse amenée à reconsidérer ses pratiques culturelles et politiques. De la même manière que le paradigme capitaliste s'est largement répandu par l'entremise des médias, le passage à un paradigme autre en l'occurrence durable - suggère, lui aussi, la nécessité d'un appui médiatique. En effet, les médias de masse (presse, télévision, Internet et réseaux sociaux) sont devenus les communicateurs incontournables des sociétés actuelles, capables d'influencer valeurs, aspirations, attentes et comportements citoyens (Hansen, 1991 ; Chomsky et Herman, 2008). Le développement durable est justement affaire de comportements. Plusieurs auteurs insistent de ce fait sur la nécessité de bâtir une culture du DD capable d'inspirer un nouvel élan dans les politiques publiques ainsi que chez les citoyens (Jégou, 2007 ; Besancenot, 2009). En véhiculant les principes du DD auprès de citoyens et de personnalités politiques qui, pour la plupart, n’ont que la société de consommation pour cadre de référence, les médias sont, de fait, des acteurs à part entière du développement durable (Nitz, 2000 ; Leal Filho et Borner, 2005).

Cet article explore les liens entre l'attention de la presse montréalaise pour le DD et l'émergence d'un cadre institutionnel dédié à la mise en œuvre du DD à Montréal. Il s'intéresse également aux interprétations du concept forgées par la scène médiatique et politique montréalaise et qui influencent nécessairement le niveau de réceptivité des citoyens aux changements de paradigme qu'il invite à réaliser.

Notre hypothèse postule que l'attention médiatique et la prise en charge institutionnelle du DD à l'échelle de la ville Montréal coévoluent. Dans la mesure où l'attention que portent les médias à divers sujets intervient sur la formation de l'opinion publique et sur l'agenda politique, comment les cycles d'attention de la presse montréalaise pour le DD s'articulent-ils par rapport au Premier plan stratégique de développement durable de la collectivité montréalaise (2005-2009)?

L'étude du lien entre agenda médiatique et agenda politique est critiquée par d'aucuns qui considèrent qu'un effet direct des médias sur les politiques publiques ne serait pas observable de manière systématique. Comme McCombs et al. (1991), ou encore Chomsky et Herman (2008), Nollet (2009: 7) remarque que ce lien causal dépend de la manière dont «le cadrage médiatique d'un problème public peut servir les intérêts des décideurs». Ceci nous amène à poser une seconde hypothèse selon laquelle le lien entre attention médiatique et action politique autour du DD ne se vérifie pas seulement à travers l'approche quantitative et chronologique des cycles d'attention médiatique. En effet, nous pensons qu'on peut l'observer jusque dans le contenu même des articles de la presse montréalaise sur le DD et des résolutions du Premier plan stratégique de développement durable de la collectivité montréalaise. Mais de quelle manière les interprétations du DD qui se dégagent de ces contenus se rejoignentelles? Autrement dit, dans quelle mesure les acteurs médiatiques et politiques de Montréal s'accordent-ils sur le sujet du développement durable?

Cet article est divisé en trois sections principales. Alors que la première section présente le cadre théorique et conceptuel, la seconde explicite la méthodologie. Quant à la troisième, elle contient l'analyse des résultats ainsi que les axes de discussion pertinents que ces résultats engagent, suscitent ou évoquent. Enfin, la conclusion revient sur les points saillants de la recherche, ainsi que sur ses limites; elle s'attache aussi à imaginer d'autres avenues de réflexion, en vue de recherches futures. 


\section{Cadre théorique et conceptuel}

\section{L'approche écosystémique du développement durable}

En 1987, la Commission mondiale sur l'environnement et le développement publie le rapport Notre Avenir à tous. Mieux connu sous le nom de Rapport Brundtland, ce document revient sur les crises plurielles qui animent les débats scientifiques, publics, médiatiques et politiques depuis la fin de la Seconde Guerre mondiale: pollutions en tous genres, explosion démographique des villes, famines chroniques, insuffisance des ressources naturelles, responsabilité des industries et des États, surenchère de l'armement mondial, etc. (CMED, 1989; McKibben, 2008). Au-delà de ces constats, déjà mis en avant notamment par le rapport Halte à la croissance? en 1972, le Rapport Brundtland propose la première définition officielle du concept de développement durable. Désormais, un développement est dit durable dès lors qu’il «répond aux besoins du présent sans compromettre la capacité des générations futures à répondre aux leurs» (CMED, 1989: 51). Cette nouvelle approche des enjeux du monde contemporain invite ainsi à un «dépassement des approches traditionnelles [en] allongeant l'horizon temporel et [en] élargissant le concept de capital» (de Perthuis, 2003: 15).

La formule du développement durable est d'emblée présentée comme la solution au bilan environnemental, économique et social alarmant de la planète, à l'aube du XXIe siècle. Il faut toutefois attendre le Sommet de la Terre de Rio de Janeiro, de 1992, pour que le terme soit consacré par les instances internationales et que ses principes commencent à se populariser (Felli, 2008). À cette occasion, la définition du développement durable (DD) est précisée par «la métaphore des trois piliers» (Flipo, 2007: 53). Selon cette approche systémique, le DD se situe à la confluence des trois piliers de la vie moderne que sont l'économie, l'environnement et la société. Il se réalise dès lors que le progrès économique est allié à la préservation de l'environnement et à la justice sociale.

Le DD est donc un concept éminemment transversal qui ambitionne de (ré)concilier le développement économique et social et la protection de l'environnement, désormais reconnue comme condition sine qua non de la survie de l'espèce humaine. L'idée sous-jacente à cette définition est que, dans la mesure où les activités humaines ne tiennent pas compte des limites des écosystèmes, le modèle capitaliste dominant actuel est, de par sa nature même, non durable (Felli, 2008; Anton, 2009).

Inhérente aux trois piliers, la notion de gouvernance qui régule les processus de prise de décision et tisse les liens entre acteurs et territoires apparaît comme un enjeu transversal, indispensable à la réalisation pratique du DD. Dans cette perspective, les principes du DD doivent trouver une place toute particulière dans le jeu des acteurs et aux différentes échelles territoriales sur lesquelles ces derniers interviennent.

Certes, la célèbre formule «Penser globalement - Agir localement» résume bien l'idée d'association des échelles territoriales du DD. Mais l'abstraction de son horizon temporel, l'absence de références spatiales précises, la complexité des rapports d'échelles et la multiplicité des acteurs impliqués rendent difficile l'articulation des ambitions globales et locales du DD. Si l'émergence du concept de DD a été motivée par les enjeux globaux d'une société-monde, il n'en reste pas moins que le territoire doit tenir une place privilégiée dans toute stratégie se réclamant de ses principes (Gumuchian et al., 2000; Jégou, 2007; Besancenot, 2009). Pour reprendre l'expression de Theys (2002: 3), le territoire est ainsi la «brique de base du développement durable». 
Se pose alors la question de savoir quelle échelle territoriale est la plus pertinente à la mise en œuvre du DD et à l'adoption par le plus grand nombre possible de citoyens sinon par tous - des comportements responsables sous-jacents à sa définition.

\title{
La ville: échelle stratégique à l'implantation du développement durable
}

Si l'histoire montre que le palier municipal n'a pas constitué le vecteur central des politiques environnementales, Juillet et Andrew (1999) remarquent toutefois qu'il existe un lien clair entre le développement durable et le discours contemporain sur le développement urbain.

\begin{abstract}
La ville ne donne pas seulement un cas d'application pratique du "développement durable», elle permet aussi de comprendre quelles peuvent être nos prises sur la dynamique de la mondialisation. [Ainsi,] la métropole est une échelle d'analyse révélant la nouvelle interrogation portant sur l'abondance et la rareté (Padis, 2009: 58).
\end{abstract}

Centre du pouvoir et véritable «symbole d'un mode de développement et d'une manière de vivre ensemble» (Baddache, 2006: 137), la ville est le lieu de l'expérimentation sociale par excellence. C'est d'ailleurs à cette échelle territoriale que se croisent les principaux enjeux du DD et que s'offrent aussi les plus grandes perspectives de son succès (CMED, 1989; Besancenot, 2009).

Aujourd'hui, le palier municipal se montre souvent plus réactif dans l'intégration des principes du DD que bien d'autres échelles de gouvernance territoriale (Wackermann, 2000). Comme le remarquent Richardson et Gordon (2001), l'adjectif durable est désormais incontournable dans toute stratégie locale de planification urbaine. De nombreuses villes à travers le monde reconnaissent les défis majeurs que la gestion durable de leur environnement urbain pose pour les décennies à venir. Proactives, elles voient dans le DD un moyen de se positionner en acteurs d'une croissance plus intelligente, quitte à devancer l'engagement politique de l'État central (Hamman, 2012) .

Les origines de la ville viable ${ }^{1}$ remontent à la fin des années 1960 avec la remise en question de la théorie classique du développement et l'émergence de l'environnementalisme contemporain. Contrairement aux modèles urbains classiques, la ville viable cherche à réconcilier milieu urbain et milieu naturel; elle envisage ainsi les préoccupations environnementales, politiques et sociales sur un pied d'égalité. Les enjeux privilégiés sont notamment: les transports en commun, la consommation responsable, l'efficacité énergétique des bâtiments publics, la sécurité, le multiculturalisme, etc. (Richardson et Gordon, 2001 ; Lafrance et Lafrance, 2014).

Les aires urbaines concentrent aujourd'hui l'essentiel des activités économiques et plus d'un habitant de la planète sur deux. En s'inscrivant en ville - «reflet privilégié de l'espace-temps» (Wackermann, 2000: 28) - il est légitime de penser que le DD est en mesure de rejoindre un nombre maximum de citoyens-acteurs et de créer ainsi les conditions optimales de son succès. En effet, si de nombreux auteurs reconnaissent

1 Nous utilisons le terme viable parce qu'il traduit mieux l'idée d'autosuffisance urbaine (sur les plans agricole, énergétique, etc.) que l'adjectif durable, aujourd'hui très galvaudé. Le Sommet de Rio de Janeiro, de 1992, a consolidé la réflexion naissante sur l'intégration des principes du DD à l'échelle urbaine. La ville viable devient un véritable mouvement avec la Charte d’Aalborg (24 mai 1994) signée par 80 villes d'Europe cherchant à traduire à l'échelle locale urbaine les principes du référentiel de planification stratégique Agenda 21 (Emelianoff et Stegassy, 2010). 
que la gouvernance du DD doit être amorcée par le niveau étatique, ils concèdent aussi qu'elle ne saurait reposer sur la seule initiative des représentants de l'intérêt général (Balthazard, 2006; Cluzet, 2010). Comme le souligne Besancenot (2009: 195), le DD doit donc commencer «par l'échelon décisionnel le plus proche de nous : le simple citoyen».

\title{
Les cycles d'attention médiatique: un baromètre de l'intégration sociale du développement durable
}

L'existence d'un lien entre l'attention médiatique accordée à un problème de société et la mobilisation de l'action publique et collective autour de la résolution de ce problème a fait l'objet de nombreuses études. Ce lien se vérifie dans le contexte particulier à la crise environnementale (Downs, 1972) ${ }^{2}$ tout comme dans celui de l'intégration sociale des principes du développement durable (Holt et Barkemeyer, 2010).

\begin{abstract}
To some planners and decision makers, the growth of the planet in a sustainable way rests as much on journalists' as it does on those of national leaders. They consider journalists not only 'public educators' but also 'social engineers' who can effectively help transform the values and ethics of society towards sustainable development (Leal Filho et Borner, 2005 : 5).
\end{abstract}

Si les médias de masse n’apportent pas directement de changements au sein de la société, ils favorisent néanmoins la réceptivité des citoyens aux différents courants idéologiques qui la traversent (Hansen, 1991; Leal Filho et Borner, 2005). Ils contribuent ainsi à placer les thèmes de l'environnement et du développement durable dans l'ordre du jour de la société. La majorité des décideurs politiques considèrent d'ailleurs que leur réflexion personnelle sur les enjeux du développement auxquels ils sont confrontés s'appuie largement sur l'information portée par les médias locaux, nationaux et internationaux (Boykoff et Roberts, 2007). En tant qu'acteurs du DD, les médias jouent un double rôle: ils agissent à la fois comme support à la conscientisation citoyenne et à l'expression des acteurs - notamment politiques - et exercent une influence sur l'opinion publique et sur les stratégies décisionnelles en matière de développement durable (Nitz, 2000).

Comme le note Flipo (2007: 9), «le développement durable réactualise la question du politique». Le concept de mise à l'ordre du jour politique est aujourd'hui reconnu comme un facteur médiatisant les rapports entre le champ des politiques publiques, la sphère des médias et l'opinion publique. Il renvoie à l'ensemble des enjeux traités par les autorités publiques, cette prise en charge prenant notamment la forme de décisions politiques (Bregman, 1989).

En jetant la lumière sur un thème particulier et en laissant d'autres thèmes dans l'ombre, les médias influencent de fait le public, qui perçoit l'importance (l'urgence) d'un enjeu en fonction de l'importance (la visibilité) que lui accordent les médias (Valenti, 2000). Comme le remarquait déjà Cohen en 1963 (dans McCombs et al., 1991: 12), s’ils ne nous disent pas quoi penser («what to think»), les médias nous disent néanmoins à quoi penser («what to think about»). Ainsi, c’est l'attention soutenue des médias et de leurs publics pour un enjeu de société particulier - et la critique du pouvoir qu'elle

2 En réponse à la pression de l'opinion publique américaine, alimentée par la couverture médiatique de plusieurs accidents industriels engageant la santé publique et par la publication de nombreux travaux scientifiques, les décennies 1960 et 1970 voient la naissance d’un important mouvement de législation environnementale (McKibben, 2008). 
sous-entend - qui peut inciter la sphère politique à modifier le statu quo à travers les politiques publiques qu'elle estime pertinentes (Nollet, 2009). En conséquence, l'ordre du jour politique se voit influencé par «l'agenda des médias et l'agenda des citoyens (les préoccupations de l’opinion publique)» (Bregman, 1989: 192).

Afin de mettre en évidence et tenter de qualifier le lien entre couverture médiatique, opinion publique et politiques publiques, un certain nombre de modèles théoriques ont été développés. Défini et appliqué à la thématique environnementale par Downs (1972), ${ }^{3}$ le modèle des cycles d'attention médiatique (CAM) démontre que le niveau de résonnance médiatique d'un thème fluctue dans le temps et qu'il influence l'opinion et les pouvoirs publics (figure 1).

Cette dynamique s'organise en cinq paliers qui apparaissent presque toujours dans le même ordre et dont la durée varie en fonction du caractère newsworthy ${ }^{4}$ de l'actualité. Le premier palier marque le coup d'envoi de la médiatisation d'un enjeu: le public est alors encore peu sensibilisé. S'ensuit le second palier, qui se caractérise par une augmentation, souvent rapide, de la couverture médiatique activée par des événements déclencheurs ${ }^{5}$ : en réaction, le public se sent investi, concerné, voire inquiet. Le point culminant de la couverture médiatique octroyée à un enjeu donné est atteint lors du troisième palier. Celui-ci va souvent de pair avec la révélation des coûts (au sens large du terme) associés au règlement de l'enjeu social soulevé et s'accompagne d'un certain sensationnalisme médiatique. Le quatrième palier est marqué par une perte progressive d'intérêt des médias au profit d'autres thèmes jugés plus urgents; le public quant à lui, tend à se résigner. Enfin, le cinquième palier correspond à un intérêt médiatique résiduel et à un désengagement relatif de l'intérêt du public. Toutefois, l'enjeu social révélé suscite maintenant une perception différente chez les citoyens. En effet, même s'il est éclipsé des contenus de presse, il est désormais inscrit à l'agenda sociopolitique de la société, du moins en paroles. Cette remarque est particulièrement valable pour les thèmes de l'environnement et du développement durable, qui refont fréquemment surface dans l'actualité. Ajoutons que le cinquième palier est souvent accompagné d'une prise en charge institutionnelle plus cohérente que les actions politiques ponctuelles généralement déployées aux paliers précédents (Downs, 1972; Holt et Barkemeyer, 2010).

Les cycles d'attention médiatique et la ville viable illustrent respectivement l'inscription du développement durable dans les agendas médiatiques et politiques des sociétés. Ultimement, les concepts d'agenda médiatique et d'agenda politique permettent d'observer si les élus et les autorités publiques tiennent compte de l'opinion publique, en partie construite, relayée et diffusée par les médias (Nollet, 2009). Dans le cadre de cette étude, nous mettons en lien agendas médiatique et politique, sans l'intermédiaire de l'opinion publique. Nous envisageons ainsi ce que Yanovitsky (2002: 422) qualifie de «Media-Policy Connection» dans la reconnaissance médiatique et institutionnelle du développement durable à l'échelle de la ville de Montréal.

3 Howlett (1997) a appliqué la théorie des CAM à l'émergence de la politique environnementale canadienne.

4 Ce terme n’a pas d'équivalent en français. Il désigne le caractère particulier d'une information considérée comme digne d'attention et valant la peine d'être médiatisée par un média donné.

5 Traduction libre de l'expression «trigger events» utilisée par Holt et Barkemeyer (2010: 7). 
Figure 1 Les médias: un pont entre $\mathrm{DD}$, opinion publique et action politique

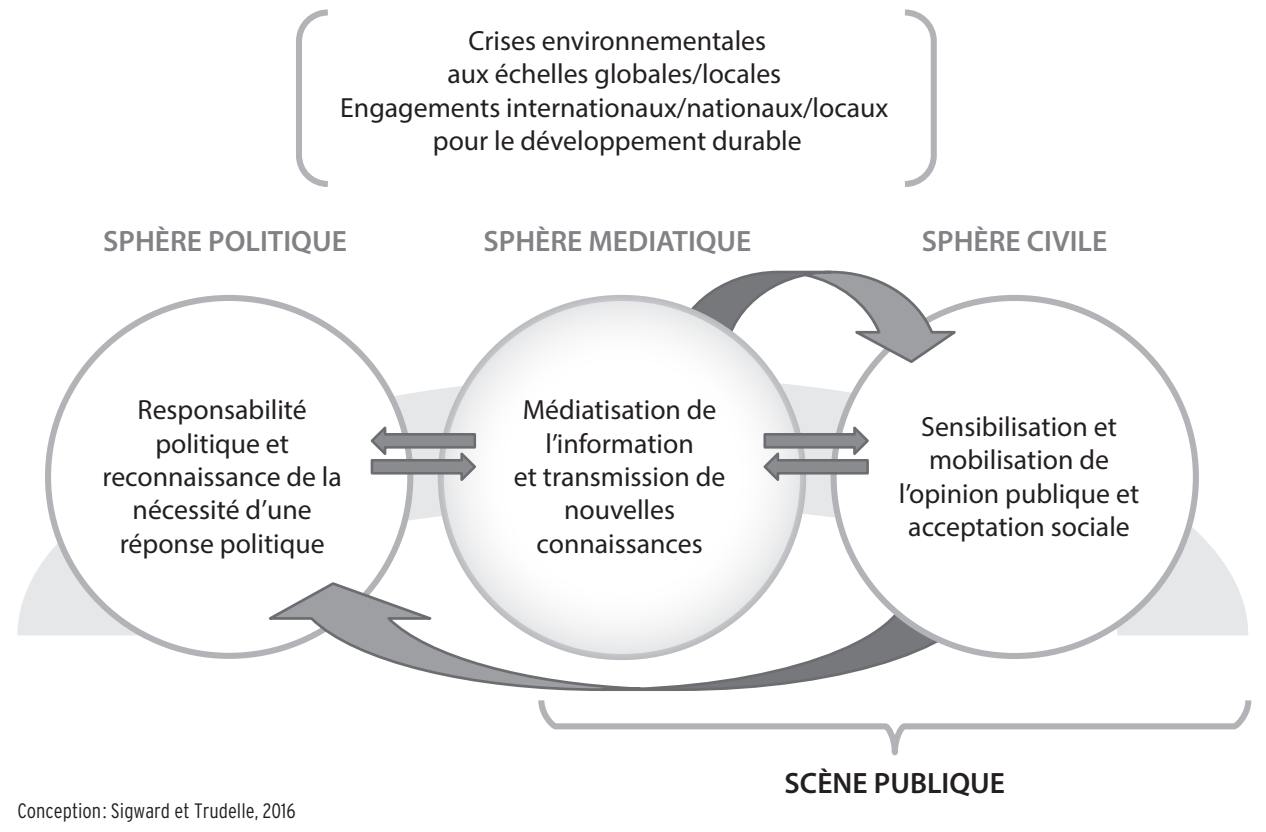

\section{Méthodologie}

\section{Cadre spatiotemporel de la recherche}

\section{Montréal: lieu d'innovations sociales et territoriales}

Montréal a démontré assez tôt un intérêt pour la question du développement durable urbain $^{6}$ en mettant en œuvre des initiatives locales à caractère environnemental et social et en soutenant de nombreux projets qu'il est aujourd'hui possible de qualifier de durables. ${ }^{7}$ Mais la réponse politique au DD est somme toute assez récente. De fait, cette réponse se matérialise sur la scène politique montréalaise le 1er avril 2005, avec l'entrée en vigueur du Premier plan stratégique de développement durable de la collectivité montréalaise (2005-2009) (Ville de Montréal, 2010; CRE, 2010).

Avec ses 1649519 habitants (Statistique Canada, 2011), Montréal cumule sensibilité environnementale, forts niveaux de culture et d'éducation, trajectoires de désindustrialisation et stratégies de renouvellement urbain. Elle partage ainsi nombre de points communs avec d'autres villes du monde, telles que Copenhague (Danemark) et Portland (États-Unis) qui ont fait le choix politique du développement durable (Richardson et Gordon, 2001; Lafrance et Lafrance 2014). Deuxième plus grande ville du Canada après Toronto, Montréal est aussi un centre médiatique important, centralisant de nombreuses entreprises médiatiques québécoises (Raboy, 2000).

6 En 1993, Montréal accueillait le quatrième congrès mondial Metropolis '93, intitulé: «Le citoyen et le développement durable» (Metropolis, 2015).

7 Citons en exemples l'initiative des Toits Verts, le projet Défi Climat, ou encore, la Convention de la Diversité Biologique à l’ONU, dont le secrétariat est à Montréal depuis 1996. 
Dans cette perspective, nous pensons que le territoire montréalais constitue un lieu propice à la médiatisation des principes du DD auprès des citoyens et des acteurs politiques, ainsi qu'à une intervention publique durable.

\section{7-2009: deux bornes chronologiques clés}

Cette étude s'étend du $1^{\text {er }}$ janvier 1987 au 31 décembre 2009. Ces deux dates s'inscrivent dans une période de forte attention médiatique et politique pour les thèmes de l'environnement et du DD, du niveau local au niveau international. En effet, si 1987 est l'année de publication du Rapport Brundtland, c'est aussi celle de la signature du Protocole de Montréal contre l'utilisation des chlorofluorocarbures (CFC). ${ }^{8}$ La fin de l'année 2009, quant à elle, est marquée par la tenue de la conférence des parties de la Convention des Nations Unies sur le Changement Climatique, de Copenhague ${ }^{9}$, et annonce la fin du Premier Plan stratégique de développement durable de la collectivité montréalaise (2005-2009).

\section{Les sources de données analysées}

\section{Une source politique: le Premier plan stratégique de développement durable de la collectivité montréalaise (2005-2009)}

Cluzet (2010:7) souligne que le développement durable n’est que «trop souvent utilisé à de seules fins commerciales ou politiciennes» et que les politiques dites durables se résument généralement à une simple liste de mesures se contentant d'inviter à de nouveaux comportements. D’aucuns remarqueront néanmoins que c'est de cette manière que la plupart des citoyens sont éduqués au développement durable.

D’après le Premier plan stratégique de développement durable de la collectivité montréalaise (PPSDDCM), ${ }^{10}$ Montréal est une métropole activement engagée sur la voie de la durabilité. L'administration municipale de Montréal et ses partenaires se sont ainsi formellement engagés «à réaliser certaines actions au sein de leurs activités corporatives ou dans leurs prestations de services auprès de leur clientèle» (Ville de Montréal, 2010: 12). Fruit de plus de trois années de réflexion entre les différents acteurs de la société montréalaise, ${ }^{11}$ le PPSDDCM contient 36 actions présentées sous forme de résolutions estampillées développement durable et

8 Composants chimiques responsables de la dégradation de la couche d'ozone, un enjeu très médiatisé tout au long de la décennie 1980. Le Protocole de Montréal est le premier protocole environnemental à obtenir une ratification universelle et à avoir une valeur contraignante (Keating, 1993; Sachsman, 2000) .

9 La COP 15 de 2009 devait renégocier les accords internationaux sur le climat (Protocole de Kyoto de 1997). Les participants signent un accord non-contraignant visant à limiter le réchauffement climatique à 2 degrés d'ici 2050 .

10 Document disponible à l'adresse suivante: http://ville.montreal.qc.ca/pls/portal/docs/page/d_ durable_fr/media/documents/bilan_2005-2009_plan_strat_dev_durable_synth.pdf

11 Au total, la mise en œuvre du PPSDDCM a réuni plus de 178 partenaires: 162 organismes externes et 16 administrations locales. Près de la moitié des partenaires sont des organismes à but non lucratif (Équiterre, l'Association québécoise pour la promotion de l'éducation relative à l'environnement (AQPERE), etc.) et un peu plus du tiers, des entreprises (notamment les quatre universités de Montréal). Les autres partenaires du Plan sont les institutions (par exemple, le ministère des Transports du Québec - Direction de l’̂̂le-de-Montréal) et les corps publics (Ville de Montréal, 2010). 
assorties d'objectifs relativement clairs, certes, mais dont les indicateurs peuvent manquer de précision. Moins circonscrit dans l'espace et dans le temps que les initiatives précédentes, le Plan est le signe d'une certaine reconnaissance politique de la nécessité d’intégrer le DD à Montréal.

\section{Une source médiatique: La Presse, The Gazette et Le Devoir}

En relatant les événements qui traversent la société à un moment précis, les journaux représentent un puissant véhicule collectif des valeurs sociales (Trudelle, 2005). Puisque l'adoption et l'intégration du DD par les sociétés contemporaines constituent précisément une question de valeurs collectives, l'utilisation de quotidiens régionaux comme source de données semble particulièrement justifiée. Pour cette étude, nous avons ainsi choisi de consulter les trois quotidiens régionaux suivants: La Presse, The Gazette et Le Devoir.

Si, aujourd'hui, la presse Internet tend à remplacer le modèle traditionnel de la presse écrite payante, l'influence de cette dernière demeure encore très forte au Québec, notamment à Montréal. Ainsi, avec plus de 1,7 million de lecteurs hebdomadaires, ces trois quotidiens représentent près de la moitié du marché de la presse quotidienne montréalaise, en 2008. Ils constituent une source d'information considérée comme sérieuse et sont fortement ancrés dans l'espace culturel montréalais, ce qui leur confère une influence sociale certaine (Raboy, 2000; CEM, 2015). Nous pensons que ces entités médiatiques représentent aussi Montréal dans sa diversité linguistique, culturelle et idéologique.

\section{Méthodes d'échantillonnage}

\section{Le corpus d'articles des cycles d'attention médiatiques (CAM)}

À partir des bases de données Eureka (pour La Presse et Le Devoir) et Canadian News Stand (pour The Gazette), nous avons constitué trois échantillons d'articles de presse (un par quotidien).

Nous avons recensé les articles en mentionnant dans le corps du texte l'expression développement durable/sustainable development (comme unité sémantique ou comme deux unités de codage distinctes) ou le terme durabilité/sustainability. ${ }^{12}$ Le nombre total d'articles retenus s'élève ainsi à 7764 (tableau 1). Précisons que tous ces articles ne traitent pas nécessairement du DD en tant que sujet d'actualité; ils peuvent n’en faire que la simple mention. Néanmoins, cela donne une bonne idée de la visibilité médiatique accordée au concept ou, à tout le moins, à l'expression développement durable.

12 Afin de déterminer ce corpus d'articles, il nous a semblé essentiel et pertinent de rechercher la présence des deux termes contenus dans l'expression développement durable. Étant donné le caractère particulièrement polysémique du terme durabilité en français, la pertinence des articles recueillis a dû être vérifiée. Aussi, après plusieurs tests sémantiques nous avons remarqué, à l'instar de Holt et Barkemeyer (2010), que The Gazette, journal anglophone, utilise de manière indifférenciée les termes sustainability (582 occurrences) et sustainable development (1250 occurrences). La Presse et Le Devoir, nos deux journaux francophones, n'utilisent que très rarement le terme durabilité (dans le sens de DD) (33 occurrences à eux deux) et emploient surtout l'expression développement durable. Par ailleurs, nous avons écarté les articles contenant les expressions développement soutenable/viable, leur nombre étant assez marginal. Ces variantes sémantiques étaient parfois utilisées, et ce, jusqu’à la parution aux Éditions du Fleuve de la troisième édition révisée francophone du Rapport Brundtland, en 1989. Dans cette version, l'éditeur précise que «l'expression développement durable semble être mieux acceptée que développement soutenable, du moins en Amérique» (CMED, 1989: xi). 
Tableau 1 Les articles recensés pour les CAM

\begin{tabular}{lcc}
\hline \multicolumn{1}{c}{ Quotidien régional } & Nombre d'articles recensés & Part de chaque échantillon de presse \\
\hline La Presse & 2697 & $34,74 \%$ \\
The Gazette & 1832 & $23,60 \%$ \\
Le Devoir & 3235 & $41,66 \%$ \\
Total & 7764 & $100 \%$ \\
\hline
\end{tabular}

\section{Le corpus d'articles destinés à l'analyse de contenus}

Afin de constituer ce second corpus d'articles, nous avons effectué la même requête en recherchant les mêmes mots-clés, mais cette fois, uniquement dans le titre. Cela a permis de dégager 163 articles traitant du développement durable comme sujet d'actualité à part entière (tableau 2).

Tableau 2 Les articles recensés pour l'analyse de contenu

\begin{tabular}{lcc}
\hline \multicolumn{1}{c}{ Quotidien régional } & Nombre d'articles recensés & Part de chaque échantillon de presse \\
\hline La Presse & 45 & $27,6 \%$ \\
The Gazette & 26 & $16,0 \%$ \\
Le Devoir & 92 & $56,4 \%$ \\
Total & $\mathbf{1 6 3}$ & $100 \%$ \\
\hline
\end{tabular}

\section{Le corpus des résolutions politiques destinées à l'analyse de contenu}

Nous considérons l'ensemble du contenu des 36 résolutions du Premier plan stratégique de développement durable de la collectivité montréalaise (2005-2009).

\section{Démarche méthodologique}

L'analyse de la presse écrite constitue l'un des principaux champs d'application de l'analyse de contenu et a, encore aujourd'hui, valeur de modèle méthodologique (Mucchielli, 2006; Leray, 2008). C'est cette technique, dans ses dimensions qualitatives et quantitatives, que nous avons utilisée. Elle nous a permis d'établir les cycles d'attention de La Presse, The Gazette et Le Devoir pour le thème du développement durable en compilant les 7764 articles retenus selon le mois et l'année de leur publication.

Dans la perspective de notre première hypothèse de recherche, ce classement a permis de dégager les tendances de l'attention de la presse échantillonnée et de voir si le Premier Plan stratégique de développement durable de la collectivité montréalaise a eu quelque effet structurant sur elle. Nous avons aussi évalué la place accordée à chacun des 3 piliers du développement durable dans l'ensemble des 36 résolutions politiques et des 163 articles de presse échantillonnés. Cela nous a permis d'apprécier l'interprétation du développement durable portée par la presse et la Ville de Montréal et d'établir des rapprochements susceptibles de valider notre seconde hypothèse de recherche. 


\section{Résultats et discussion}

\section{Introduction, installation et essor médiatique du thème du développement durable}

D’après nos résultats, en l'espace de deux décennies l'expression développement durable/sustainable development et le terme durabilité/sustainability se sont clairement installés dans les contenus de La Presse, The Gazette et Le Devoir. Quasi inexistants dans le vocabulaire de ces trois quotidiens avant le mois de juin 1987, ${ }^{13}$ ces termes sont couramment utilisés à l'issue de la période étudiée. De seulement deux articles dénombrés en 1987 (ce qui est le minimum), la couverture de presse du DD passe ainsi à 991 articles en 2007 (ce qui est le maximum) (figure 2).

Figure 2 Evolution annuelle de la couverture de presse montréalaise du développement durable (1987-2009)

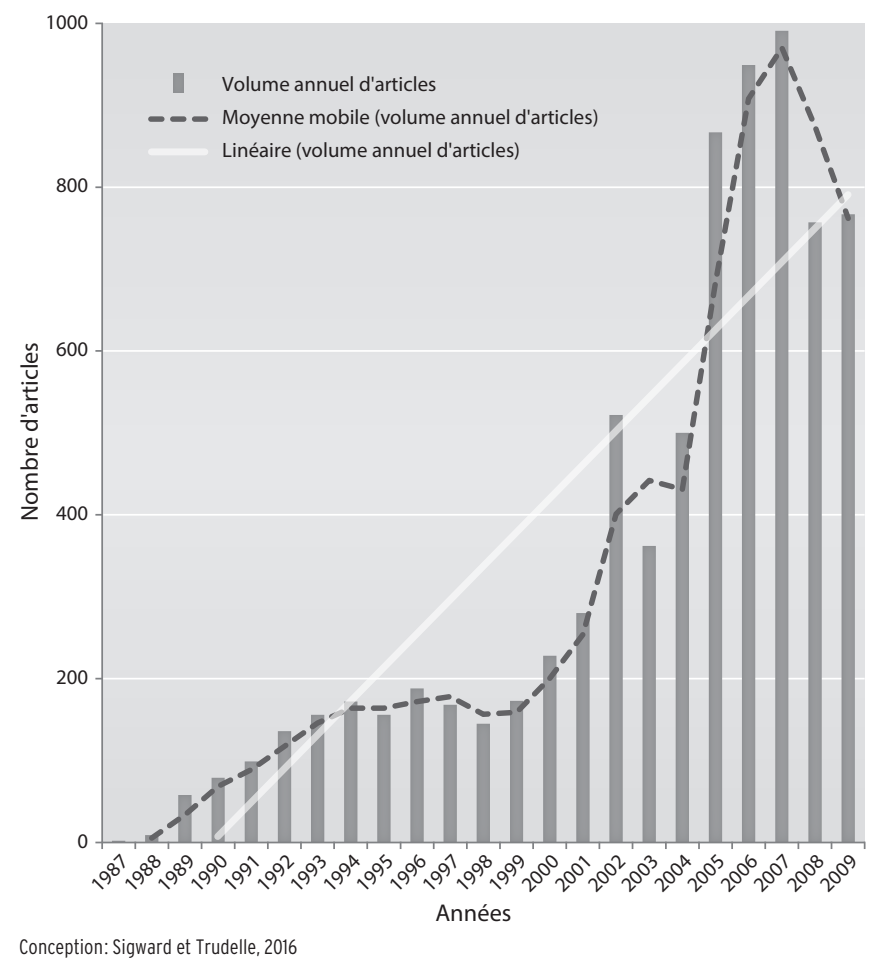

S'il apparaît que la couverture de presse montréalaise du DD progresse sur l'ensemble des 23 années de la période étudiée, ${ }^{14}$ cette trajectoire ascensionnelle n'est pas sans ponctuations, ruptures et discontinuités, notamment pour les années 1995, 1997, 1998, 2003 et 2008 (figure 2). Ces résultats nous semblent particulièrement

13 Une recherche complémentaire dans les bases de données a révélé qu'avant 1987 ces termes sont absents des contenus éditoriaux, du moins dans l'acception qu'on leur connaît aujourd'hui.

14 La moyenne mobile dégage les grandes tendances de la couverture de presse. La courbe de tendance linéaire montre l'accélération quantifiée du volume annuel d'articles recensés. 
révélateurs du fonctionnement médiatique décrit par Downs (1972), McCombs et al. (1991), ou encore par Chomsky et Herman (2008), selon lequel les médias sont capables d'imposer (et d'écarter) très rapidement une idée - ainsi que le verbatim qui y est associé - dans l'espace public.

Les années 2005, 2006 et 2007 sont celles qui ont vu La Presse, The Gazette et de Le Devoir accorder le maximum d'attention au thème du développement durable; elles réunissent à elles seules 2807 articles, soit plus du tiers de la couverture de presse totale du DD. Après l'apogée médiatique de 2007 (991 articles), l'année 2008 enregistre une diminution relativement importante $(-23,61 \%)$, tandis que l'année 2009 enregistre à nouveau une très légère progression $(+1,32 \%)^{15}$ (figure 2$)$.

\section{Événements déclencheurs et caractère cyclique de la couverture de presse montréalaise du DD}

Sur les 276 mois de l'étude, la couverture de presse montréalaise du DD s'organise en une succession de vagues, de stagnations et de creux qui se maintiennent généralement entre trois et cinq mois. ${ }^{16}$ Ce comportement général s'inscrit pleinement dans l'organisation des paliers particulière aux cycles d'attention médiatique (CAM) décrite précédemment.

Il est possible de remarquer que les variations de l'attention de la presse correspondent avec un certain nombre d'événements particuliers qui agissent en véritables déclencheurs d'attention (figure 3). Prenons d'abord l'exemple du Sommet de la Terre de Rio de Janeiro qui s'est tenu en juin 1992. Nos résultats montrent que la somme des articles abordant le DD publiés en juin (36 articles) dépasse pour la première fois la moyenne mensuelle de la période 1987-2009 établie à 28,13 articles. ${ }^{17}$ Ce premier pic d'attention semble pertinent dans la mesure où il est généralement admis que ce sommet international a joué un rôle crucial dans l'inscription du DD aux agendas politiques et médiatiques des pays occidentaux (Sachsmann, 2000; SustainAbility, 2002).

Entre 1993 et 1999, la variation annuelle du nombre d'articles reste relativement faible (figure 3). Cette observation rejoint le constat de Lewis (2000: 251) pour qui le milieu des années 1990 correspond à un issue-attention roller coaster à l'égard de la thématique du développement durable.

Avec la signature du Protocole de Kyoto, 18 en décembre 1997, il aurait été légitime de s'attendre à une plus forte résonance médiatique du thème que celle qui se dégage de nos résultats (figure 3). L’inertie des débats nationaux et internationaux autour de la question des changements climatiques, de même que la récession économique

15 Pourcentages d'évolution obtenus à partir de la formule: (valeur d’arrivée - valeur de départ/valeur de départ)*100.

16 Autre remarque importante dans la mesure où elle vient valider la pertinence des mots-clés utilisés dans le dénombrement des articles sélectionnés: La Presse, The Gazette et Le Devoir enregistrent des tendances très similaires en regard de l'attention qu'ils accordent au DD, que ce soit en termes de volume d'articles ou de temporalité.

17 Résultat obtenu par la formule: nombre total d'articles recensés dans l'étude/nombre de mois concernés par l'étude.

18 Accord international visant la réduction des émissions de gaz à effet de serre responsables du réchauffement climatique. 
de 1995, peuvent en partie expliquer l'effet incertain et diffus dans le temps de la signature du Protocole sur la couverture montréalaise du DD. Selon l'étude de Holt et Barkemeyer (2010), Kyoto a d'ailleurs plus alimenté la couverture médiatique des changements climatiques que celle du développement durable. ${ }^{19}$

On aurait également pu s'attendre à une plus grande résurgence du nombre d'articles avec le passage à l'an 2000 et les bonnes résolutions internationales qui l'ont accompagné. ${ }^{20}$ En effet, l'horizon temporel du troisième millénaire a joué pour beaucoup dans la caractérisation de l'urgence d'agir en matière de protection environnementale et dans la nécessité de réinventer les modalités de développement des sociétés humaines.

Cependant, d'après nos résultats il faut attendre le second semestre 2002 pour observer un véritable regain d'attention de la presse montréalaise pour le DD. Ainsi, le mois de juin 2002 compte 56 articles, contre 23 en avril et 40 en mai. Cette résurgence du nombre d'articles peut s'expliquer par la tenue du Sommet de Montréal ${ }^{21}$ les 4, 5 et 6 juin 2002. Seuls 24 articles sont dénombrés en juillet 2002, mais un nouveau pic d'attention très important se produit le mois suivant, en août 2002. Celui-ci correspond avec la tenue du Sommet de la Terre de Johannesburg (du 26 août au 4 septembre 2002). Les nombres d'articles recensés en août et en septembre sont respectivement de 120 et 74 , alors qu'ils ne sont que de 24 en juillet (avant Johannesburg) et de 33 en octobre (après Johannesburg), avant de retomber à seulement 19 articles en fin d'année (figure 3).

Même si la couverture de presse du DD connaîtra des ralentissements par la suite, il semble que le Sommet de Johannesburg ait contribué à l'installation durable du thème du DD dans la presse montréalaise (figure 3). Plusieurs recherches corroborent cette observation et font du Sommet de Johannesburg un événement déterminant dans la mise à l'agenda politique international du DD et dans la mise en relation des sphères politique, médiatique et citoyenne autour de ses enjeux et de sa mise en œuvre pratique (SustainAbility, 2002; Holt et Barkemeyer, 2010).

19 En 2005, nos données font état de 66 articles recensés en octobre, 124 en novembre et 70 en décembre. Il est ainsi difficile d'évaluer l'effet éventuel de la tenue de la COP 11 à Montréal en 2005 sur notre couverture de presse, la rencontre ayant eu lieu entre le 28 novembre et le 9 décembre de cette même année.

20 En 2000, dans le cadre du plus grand rassemblement de dirigeants étatiques jamais organisé, les Nations Unies se sont fixé huit Objectifs du Millénaire pour le développement à atteindre pour 2015 (http://www.un.org/fr/millenniumgoals/).

21 Qui a d’ailleurs posé le premier jalon du Premier plan stratégique de développement durable de la collectivité montréalaise (2005-2009). 
Figure 3 Événements déclencheurs et niveaux d'attention médiatique (2001-2009)

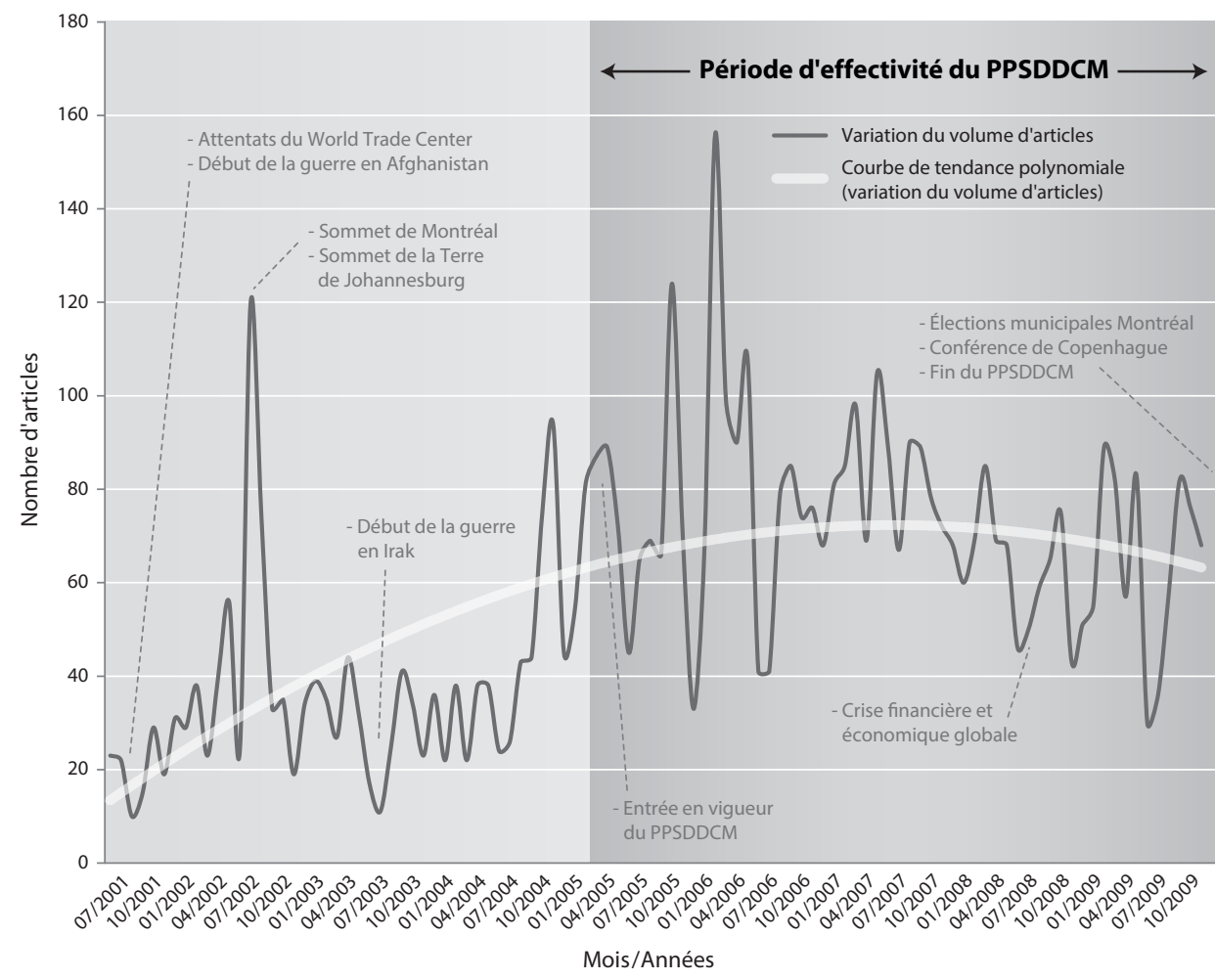

La courbe de tendance polynomiale montre la fluctuation des données dans le temps et illustre la relation entre le comportement de la couverture de presse du DD et la survenance des événements déclencheurs d'attention et d'inattention médiatique, avant et pendant le Plan. Conception: Sigward et Trudelle, 2016

Selon la théorie des CAM, certains événements captent l'attention des médias au détriment d'autres événements; ils activent un cycle d'attention qui leur est propre et qui éclipse les thèmes privilégiés jusqu'alors (Downs, 1972). C'est ce qu'indiquent nos résultats. Ainsi, les années 1990 et 1991 enregistrent une progression laborieuse du nombre d'articles abordant le DD (figure 2). Selon le rapport SustainAbility (2002), cette période coïncide avec la fin de la seconde vague environnementaliste (1988-1991) 22 et le début d'une période de désintérêt politique à l'égard du DD, amorcée avec la guerre du Golf (1991) et la récession économique des années 1990. L'étude de Lewis (2000) tend à faire le même constat: quand l'actualité économique et géopolitique mondiale prend le devant de la scène, le DD est moins médiatisé.

Comme on peut le voir sur la figure 3, cela se vérifie avec les attentats du World Trade Center, le 11 septembre 2001. Ce mois de septembre n'enregistre que 10 articles abordant la thématique du DD. Ce nombre remonte très lentement à partir d'octobre (15 articles), jusqu'au pic d'attention d'août 2002, activé par le Sommet de la Terre Johannesburg (120 articles). Mais la situation géopolitique mondiale (guerres de 2003 en Afghanistan et en Irak, toujours au premier plan de l'ordre du jour politicomédiatique, éclipse à nouveau le DD, qui ne connaîtra de véritable résurgence médiatique qu’à la fin de l’année 2004.

22 Cette vague s'est développée par la prise de conscience globale des enjeux posés par la disparition de l'ozone et de la forêt vierge, souvent présentée -par les médias notamment - comme le poumon de la planète. 
D’un point de vue quantitatif, il est intéressant de remarquer que les volumes d'articles de 2002 (522 articles) et de 2004 (500 articles) sont quasi identiques (figure 2). Ainsi, nous pensons que le Sommet de Johannesburg a contrecarré la baisse d'attention enclenchée par le climat géopolitique international très instable du début des années 2000. Étant donné nos résultats, force est de constater qu’au début de 2005 un ou plusieurs événements déclencheurs ont donné un souffle nouveau à la couverture de presse montréalaise du développement durable.

\section{Discussion 1}

\section{La place du Plan stratégique dans les cycles d'attention médiatique analysés}

Plus d'un article sur deux ${ }^{23}$ a été publié entre le début de 2005 et la fin de 2009, le volume médian de la couverture de presse survenant entre les mois de juin et juillet 2005 (figure 2). Il est ainsi possible d'observer une augmentation de la fréquence de parution des articles entre 2005 et 2009 . Cela confirme que cette période est celle où la médiatisation du DD bat son plein.

Ce constat nous invite à considérer la possibilité d'un effet structurant du Plan stratégique sur le comportement de notre couverture de presse. En effet, la période de mise en œuvre de ce programme s'étend justement du $1^{\mathrm{er}}$ avril $2005 \mathrm{au}$ 31 décembre 2009. Il est donc possible d'observer une proximité temporelle entre la mise en œuvre de la politique urbaine de DD de la Ville de Montréal et les forts niveaux d'attention de la presse montréalaise à l'égard du DD enregistrés au cours de ces mêmes années. ${ }^{24}$

Les figures 2 et 3 indiquent que l'attention médiatique relevée en 2008 est à la baisse. Nous attribuons cette tendance à la très forte médiatisation de la crise économique et financière globale de $2008^{25}$ ainsi qu'à une possible saturation médiatique du thème du DD faisant suite aux trois années de très forte couverture médiatique qui ont précédé.

À la fin de la période étudiée, on observe que le mois de décembre 2009 compte seulement 68 articles - La Presse (19), The Gazette (16) et Le Devoir (33) -, soit à peine plus que la moyenne mensuelle de l'année 2009 (63,9 articles). Cette baisse peut s'expliquer en partie par la tenue de la Conférence sur les changements climatiques de Copenhague et les élections municipales de Montréal, qui ont capté une large part de l'attention de la presse montréalaise à la fin de l'année 2009.

23 55,78\% des 7764 articles dénombrés.

24 Il est à noter que la Loi sur le développement durable (échelle québécoise), de 2006, peut avoir exercé une influence sur le comportement de la couverture de presse.

25 Selon nos résultats, le nombre mensuel d'articles recensés commence à baisser progressivement à partir du deuxième semestre 2007 (coïncidant ainsi avec la crise des subprimes ayant préfiguré la récession) pour n'atteindre que 43 articles au moment de la crise bancaire et financière de l'automne 2008. Si cette moyenne mensuelle d'articles est supérieure à la moyenne des 23 années de la période étudiée (28,1 articles), elle est en revanche bien en-dessous de la moyenne mensuelle de la forte période d'attention médiatique 2005-2009, établie à 72,75 articles. 
Dans le prolongement de la théorie des cycles d'attention médiatique de Downs (CAM) (1972), il est possible d'inférer que le relâchement de l'attention de la presse observé en 2008-2009 s'inscrit dans le mouvement d'institutionnalisation du DD enclenché par l'entrée en vigueur du PPSDDCM. Cette baisse est inhérente au modèle même des CAM selon lequel prise en charge institutionnelle et désintérêt médiatique vont de pair.

Nous pensons ainsi que le Plan a permis de contrer l'inertie et la désorganisation politiques des débuts et que la thématique du DD est devenue moins newsworthy, puisque moins conflictuelle et donc moins sujette à servir le penchant sensationnaliste des médias. Cette dynamique se traduit par la baisse constatée du nombre d'articles (figures 2 et 3 ).

Ainsi, nos résultats semblent indiquer que 1) l'attention médiatique maximum accordée au DD par nos trois journaux montréalais entre 2005 et 2007, 2) les dates de début et de fin de la mise en œuvre du PPSDDCM et 3) les baisses d'attention médiatique de 2008 et 2009 correspondent dans le temps les unes avec les autres.

\section{Prédominance médiatique des piliers économie et environnement}

Sur l'ensemble de la période 1987-2009, ce sont les piliers économie et environnement du DD qui semblent dominer les contenus de La Presse, The Gazette et Le Devoir (tableau 3).

\section{Tableau 3 La part des thèmes/piliers du DD dans les contenus de presse}

\begin{tabular}{|c|c|c|c|}
\hline Piliers et thèmes du DD & $\begin{array}{l}\text { Nombre de citations } \\
\text { sur } 163 \text { articles }\end{array}$ & $\begin{array}{c}\text { Nombre de citations } \\
\text { par pilier }\end{array}$ & $\begin{array}{l}\text { Représentation du } \\
\text { pilier (en \%) }\end{array}$ \\
\hline \multicolumn{4}{|l|}{ Économie } \\
\hline Croissance économique & 34 & & \\
\hline Surexploitation des ressources & 54 & 172 & $35,8 \%$ \\
\hline Nouvelles pratiques & 48 & & \\
\hline Coûts, investissements, politiques & 36 & & \\
\hline \multicolumn{4}{|l|}{ Environnement } \\
\hline Changements climatiques & 31 & & \\
\hline Pollution et catastrophes & 30 & 163 & $34,0 \%$ \\
\hline Protection et grands enjeux & 64 & & \\
\hline Politiques environnementales & 38 & & \\
\hline \multicolumn{4}{|l|}{ Société } \\
\hline Générations futures & 20 & & \\
\hline $\begin{array}{l}\text { Éducation et changements des } \\
\text { mentalités }\end{array}$ & 43 & 145 & $30,2 \%$ \\
\hline Grands enjeux sociaux & 48 & & \\
\hline Politiques de protection sociale & 34 & & \\
\hline Total & 480 & 480 & $100 \%$ \\
\hline
\end{tabular}

Nombre d'auteurs regrettent, voire s'inquiètent, du fait que les questions sociales soient largement absentes des discours politique et médiatique associés au DD (Brunel, 2008; Besancenot, 2009; Mancebo, 2010). Pourtant, d'après nos résultats, le pilier société du DD est loin d'être sous-représenté dans la presse montréalaise 
puisqu'il concerne 30,2\% des contenus des 163 articles échantillonnés. À cela, il faut toutefois ajouter que, à l'exception de quelques rares articles pour l'essentiel parus dans Le Devoir, le DD n'est que très peu envisagé par la presse montréalaise sous le seul angle de son pilier social, contrairement aux piliers économie et environnement.

Pour chacun des trois piliers du DD, ce sont plus précisément les thèmes de la protection de l'environnement - entendue au sens large - (64 citations), les conséquences économiques de la dégradation des ressources naturelles (54 citations) et les conséquences sociales du développement actuel (48 citations) qui prédominent (tableau 3). Les enjeux de la biodiversité sont abordés avec une certaine constance à partir du milieu de la décennie 1990. Le thème de la responsabilité sociale des entreprises (RSE) en lien avec des pratiques de production et de consommation durables n'apparaît véritablement dans la presse montréalaise qu'à partir de 2002. Rappelons que la question de l'intégration du DD dans la sphère économique a été mise en avant de manière particulièrement significative lors du Sommet de la Terre de Johannesburg, de 2002.

Jégou (2007: 6) considère tout un éventail d'acceptions du concept de développement durable. Apparue en 2002 (lors du Sommet de Johannesburg), l'acception large est alors très courante et renvoie à toutes les acceptions possibles du DD; elle est souvent celle «des tenants d'une priorité au social [et privilégie] le sort de l'humanité sur celui de la planète» (Ibid.). La diversité avec laquelle La Presse, The Gazette et Le Devoir traitent aujourd'hui du DD nous invite à considérer que c'est cette acception large du DD qui domine l'ensemble de la couverture dégagée.

Notre analyse révèle que plusieurs articles explorent le sujet du DD de manière un peu moins conventionnelle que d'autres. C'est le cas notamment des articles intitulés «Le point de vue féminin sur le développement durable» (Le Devoir, 21 janvier 2005), ou encore "Health issue is one of sustainability» (The Gazette, 27 juillet 1998). Plus originaux dans leur tonalité, il est possible de penser que ces articles reflètent aussi l'évolution de l'intégration sociale d'un concept longtemps considéré comme scientifique et technique, et donc difficilement médiatisable.

Pour Keating (1993), la couverture médiatique accordée à l'environnement est devenue plus complexe avec l'introduction du terme développement durable. Lorsque cette expression est apparue dans le vocabulaire courant, elle a souvent été comprise comme un concept rigide, ne concernant que la conservation des ressources naturelles. Sa complexité a été largement sous-estimée par les médias et, plus largement, par la majorité des acteurs qui s'en réclament (Leal Filho, 2000). Pour Valenti (2000), la manière dont les sphères économique et politique s'approprient le concept alimente un certain scepticisme, tant de la part des médias que du public. L’impression générale est que le DD cache nécessairement des intérêts particuliers. À cet égard, l'article intitulé «Le développement durable vise-t-il à sauver la «planète des riches »? » (La Presse, 18 juin 1993) illustre bien ce point. Il en va de même pour l'article intitulé «Retombée de l'Écosommet: Québec créera une organisation du développement durable» (Le Devoir, 28 juin 1996), qui souligne la récupération des discours liés au DD par les sphères politique et économique. Enfin, un article paru dans The Gazette le 2 mai 1994 porte ce titre très évocateur: "Sustainable development sounds a bit like diet ice cream». 
Pour Brune (1996: 100), les déclinaisons de l'expression développement durable («investissement éthique», "capitalisme à visage humain», «commerce équitable») sont des «oxymores et tautologies» qui augmentent la visibilité médiatique du concept de DD autant qu'ils le vident de son sens. Pour Leal Filho (2000), notamment, la diversité des emplois de l'expression développement durable et le caractère dynamique du concept de DD rendent difficile l'établissement d'une définition monolithique qui pourrait être utilisée par tous les acteurs et dans toutes les situations.

D’après la classification des piliers privilégiés par les acteurs, telle que définie par Jégou (2007), la presse montréalaise s'inscrit dans une interprétation du développement durable à mi-chemin entre celle des acteurs politiques (priorité à l'économie) et celle des écologistes (priorité à l'environnement).

\section{Surreprésentation politique du pilier environnement}

D’après notre analyse, 23 des 36 résolutions du Premier plan stratégique de développement durable de la collectivité montréalaise revêtent un caractère strictement environnemental, cinq sont d'ordre économique et aucune résolution n'est à proprement parler centrée sur le seul pilier social du DD. Cependant, certaines résolutions sont mixtes et rejoignent ainsi les dimensions relatives aux autres piliers (tableau 4).

\section{Tableau 4 La part des thèmes/piliers du DD dans les résolutions du Plan stratégique}

\begin{tabular}{|c|c|c|c|}
\hline $\begin{array}{c}\text { Piliers et thèmes } \\
\text { du DD }\end{array}$ & No des résolutions concernées & $\begin{array}{c}\text { Nombre de } \\
\text { résolutions par pilier }\end{array}$ & $\begin{array}{c}\text { Représentation du } \\
\text { pilier (en \%) }\end{array}$ \\
\hline \multirow{6}{*}{ Environnement } & $2.1 / 2.2 / 2.3 / 2.4 / 2.5 / 2.6 / 2.7$ & \multirow{6}{*}{34} & \multirow{6}{*}{$58,6 \%$} \\
\hline & $2.8 / 2.9 / 2.10 / 2.11 / 2.12 / 2.13 / 2.14$ & & \\
\hline & $2.15 / 2.16 / 2.17 / 2.18$ & & \\
\hline & $2.19 / 2.21 / 2.22 / 2.23 / 2.24$ & & \\
\hline & $2.25 / 2.26 / 2.27 / 2.28 / 2.29$ & & \\
\hline & $2.31 / 2.32 / 2.33 / 2.34 / 2.35 / 2.36$ & & \\
\hline \multirow{3}{*}{ Économie } & $2.2 / 2.9 / 2.10 / 2.15 / 2.19$ & \multirow{3}{*}{13} & \multirow{3}{*}{$22,4 \%$} \\
\hline & $2.20 / 2.21 / 2.22 / 2.24$ & & \\
\hline & $2.30 / 2.27 / 2.29 / 2.35$ & & \\
\hline \multirow{2}{*}{ Société } & $2.5 / 2.10 / 2.13 / 2.15 / 2.22 / 2.24$ & \multirow{2}{*}{11} & \multirow{2}{*}{$19 \%$} \\
\hline & $2.26 / 2.27 / 2.29 / 2.30 / 2.34$ & & \\
\hline Total & & 58 & $100 \%$ \\
\hline
\end{tabular}

En privilégiant l'environnement à 58,6\%, le PPSDDCM ne s'inscrit pas dans l'acception médiane du DD, qui place les trois piliers du concept sur un pied d'égalité. Il s'éloigne aussi de la vision classique des politiciens dépeinte par Jégou (2007: 8), marquée par une "prépondérance [d'] objectifs économiques» qui englobent les sphères sociale et environnementale. En fait, le Plan semble plutôt s'inscrire dans la vision des écologistes. Cette vision met l'accent sur des objectifs environnementaux englobant les piliers social et économique du DD. De prime abord surprenant, ce constat peut s'expliquer par le fait que le Plan stratégique soit appuyé sur la participation de nombreux acteurs de la société montréalaise, dont plusieurs grandes organisations 
non-gouvernementales (ONG) environnementales. Le document Bilan synthèse 20052009 du PPSDDCM (Ville de Montréal, 2010: 12) reconnaît d'ailleurs le «caractère environnemental du Plan». On remarquera aussi que c'est de la grande région de Montréal que proviennent la majorité des émissions de gaz à effet de serre (GES) du Québec. Cela peut justifier, en partie, l'attention particulière du PPSDDCM pour l'environnement et la question des changements climatiques.

Si l'on s'intéresse aux articles qui traitent spécifiquement du PPSDDCM en tant que sujet d'actualité, on s'aperçoit que ce sont ses résolutions à caractère environnemental qui sont le plus souvent mises en avant, notamment les résolutions 2.4 (utilisation du vélo en ville), 2.7 (réduction des GES), 2.17 et 2.18 (axées sur la collecte et le traitement des matières résiduelles). Cela rejoint les propos de Baddache (2006), ou encore de Brunel (2008) pour qui les discours alarmistes des médias et les solutions et gestes citoyens dont ils font la promotion rappellent le citoyen à l'ordre et lui permettent - surtout - de se décharger de sa culpabilité de consommateur. Ainsi, en s'intéressant plus volontiers aux résolutions du PPSDDCM renvoyant au pilier environnement du DD, la presse montréalaise semble valider la couleur environnementale du Plan, et donc l'acception rétrécie du DD privilégiée par les autorités publiques locales montréalaises.

\section{Discussion 2}

\section{Vers un rapprochement des contenus médiatique et politique du DD}

Si nous pouvons rapprocher dans le temps les cycles d'attention de la presse et l'action de la Ville de Montréal, un rapprochement des contenus médiatique et politique produits semble, quant à lui, moins aisé.

Nous avons vu que, sur les 23 années de l'étude, la presse montréalaise opte pour une interprétation large du DD. Mais cette interprétation n'est pas rigide et suit l'évolution de l'acception du DD dans le temps. L'interprétation du DD qui se dégage du Premier plan stratégique de développement durable de la collectivité montréalaise correspond à l'acception rétrécie du DD. Cette interprétation est nécessairement plus fixe.

Toutefois, si l'on ne s'intéresse qu'aux articles parus pendant la mise en œuvre du PPSDDCM (2005-2009), alors les représentations médiatique et politique dégagées convergent d'avantage (figure 4). En fait, il semblerait que les contenus médiatiques tendent à s'inscrire dans l'acception rétrécie du DD, acception privilégiée par le PPSDDCM et dans la grande majorité des discours et politiques durables actuels. Cette acception se focalise sur les changements climatiques, la responsabilité sociale des entreprises et l'écocitoyenneté (Jégou, 2007; Brunel, 2008; Hamman, 2012). Nos résultats montrent que, dans les dernières années, les contenus de la presse montréalaise privilégient ces thèmes tout en conservant une compréhension tridimensionnelle du concept. Le Premier plan stratégique de développement durable de la collectivité montréalaise (2005-2009), quant à lui, fait de ces thèmes les points centraux, pour ne pas dire exclusifs du contenu de ses 36 résolutions. ${ }^{26}$

26 Cela se vérifie, par exemple, dans les intitulés des résolutions 2.1, 2.5, 2.6, 2.7, 2.8, 2.17, 2.18 et 2.25 du Plan. 
En termes quantitatifs, la représentation quasi équilibrée des 3 piliers du DD observée par la presse montréalaise évolue peu entre les 18 années précédant le PPSDDCM et les 5 années de sa mise en œuvre. Toutefois, d'un point de vue qualitatif, il est possible de noter la réaffirmation de la question économique du $\mathrm{DD}$, ainsi qu'un recentrage des contenus de presse autour de sa dimension sociale. Cette évolution semble s'être faite au détriment du pilier environnement.

Jégou (2007 : 7) remarque que «L'acception rétrécie [du DD] correspond à l'obsession de la lutte contre le changement climatique, qui tend à assimiler le développement durable à celui-ci» et souligne que cette acception «relève plus d'un récent effet de mode et d'une forte volonté politique». Sur les 163 articles de presse analysés, 31 articles (La Presse [10], The Gazette ([10], Le Devoir [11]) abordent avec insistance la question des changements climatiques. Ces articles sont, pour l'essentiel, parus après 2002. Cela corrobore le constat de Brunel (2008) pour qui le vaste concept du développement durable s'est concentré, dans les médias notamment, sur le thème des changements climatiques à la suite du Sommet de la Terre de Johannesburg, en 2002.

Nous l'avons dit, étant donné l'interprétation trop souvent partielle des acteurs, la médiatisation et l'institutionnalisation du DD ne vont pas sans mettre en question la faculté des médias de vulgariser les principes parfois techniques du DD auprès des citoyens et la capacité des politiques publiques urbaines dites durables, d'en organiser et d'en assurer la mise en œuvre, sans en dénaturer le sens et la portée (Keating, 1993 ; Balthazard, 2006). Notre analyse de contenu montre que, surtout à partir de la décennie 2000, les articles ont tendance à adopter une position protectrice à l'égard du développement durable. Tels des gardiens de sa définition, ils n’hésitent pas à jeter un regard critique sur les dérives politiques (surtout aux échelles canadiennes fédérale et provinciale) associées à la mise en œuvre du développement durable.

S’il est vrai que le développement durable est avant tout un concept économique, 27 rappelons que sa finalité reste le bien-être des individus, aujourd'hui et demain. L'analyse de contenu de notre échantillon de 163 articles révèle près d'une vingtaine d'articles faisant preuve d'un certain sens critique à l'égard du paradigme de la croissance continue. Ces articles semblent mettre un point d'honneur à (re)définir l'expression développement durable. Près d'un article sur quatre revient, même sommairement, sur la définition conceptuelle du développement durable. Comme Lewis (2000), nous remarquons que ces articles utilisent systématiquement la définition des trois piliers, ou une variante proche. Une bonne partie de ces articles est parue au début de la décennie 1990 et de manière encore plus prégnante après le Sommet de Johannesburg de 2002. L'article paru le 16 août 2002 dans La Presse et intitulé «Qu'est-ce-que le développement durable? » peut ainsi illustrer le choix du journal de revenir sur un concept connu de tous, certes, mais encore mal compris. Au même moment, Le Devoir publiait un article à l'angle d'approche très similaire:

27 Dans la mesure où ses fondements reposent sur un questionnement autour de la dualité de l'enjeu croissance-décroissance. 
«Développement durable: le temps d'agir» (31 août 2002). Il semble que ce soit dans ces articles aux accents pédagogiques que la tridimensionnalité conceptuelle du DD soit la mieux intégrée et expliquée. Leurs auteurs endosseraient ainsi le rôle d'éducateur du lecteur-citoyen (et acteur potentiel du DD), rôle notamment avancé par Keating (1993) et Leal Filho et Borner (2005). ${ }^{28}$

Les articles qui offrent un point de vue critique sur le DD s'attaquent plus à la compréhension du concept par les acteurs politiques ${ }^{29}$ et économiques qu'à la pertinence du concept même. D’ailleurs, au terme de la période étudiée, les articles semblent défendre le concept contre la mauvaise interprétation de certains acteurs de la société; c'est notamment le cas des articles «Greenpeace: Charest travestit l'idée de développement durable» et «Le développement durable, une idée souvent déformée», publiés par Le Devoir (le 15 mars 2006 et le 30 mai 2007). Ce constat tend à confirmer les conclusions de l'étude de Lewis (2000) réalisée à l'échelle de la presse étasunienne et qui soutient que c'est l'interprétation des acteurs les plus puissants du DD (entreprises et ONG environnementales en tête) qui domine le contenu des médias de masse. Ces acteurs mettent ainsi en avant le pilier du développement durable qui concerne leur sphère d'activité particulière et délaissent plus ou moins les deux autres.

Toujours selon Lewis (2000), le traitement médiatique du DD manquerait ainsi de diversité dans ses points de vue idéologiques et opterait pour une vision du concept qui ne serait finalement que l'extension du discours classique sur le développement, entendu au sens de croissance continue. Nos résultats montrent d'ailleurs que ce sont les piliers économie et environnement qui sont les plus souvent associés. Ainsi, dans l'article «Développement durable: l'impasse économique» (Le Devoir, 28 février 2008), si la définition tridimensionnelle du DD est bien expliquée, c'est pourtant l'économie qui est présentée comme le levier permettant la mise en œuvre du DD. Dans le même ordre d'idées, l'analyse de contenu révèle une dizaine d'articles abordant essentiellement les gains que le DD est susceptible de générer pour la sphère économique (par exemple, «Le développement durable payant», [La Presse, 19 décembre 2006], ou encore «Business world discovers sustainability can pay» [The Gazette, 3 juillet 2008]).

Sans rien enlever à la pertinence des résolutions environnementales affichées par le Plan, il peut être dit que la question économique ne semble jamais très loin. Par exemple, la résolution 2.2 (sur la réduction du stationnement dans le centre-ville de Montréal), que nous avons classée dans le pilier environnement, renvoie aussi au pilier économie dans la mesure où il est possible d'arguer qu'elle traduit aussi une intention de réappropriation de l'espace foncier urbain par la Ville de Montréal (tableau 4).

Ce second rapprochement des contenus vient donc nuancer les résultats qualitatifs de la représentation médiatique du DD observée dans les journaux à l'étude. En effet, il nous semble qu'en privilégiant la dimension environnementale du Premier plan stratégique de développement durable de la collectivité montréalaise, la presse montréalaise s’inscrit

28 Notons que plus de $15 \%$ des 163 articles analysés ont ainsi été rédigés de la main d'experts. Notamment: Jean-Louis Bourque, politologue (La Presse), Peter Calamai, journaliste spécialiste des sujets scientifiques et Jay Bryan, chroniqueur régulier de la section Business and Economics (The Gazette), Louis-Gilles Francoeur, spécialiste de la thématique environnementale, Corinne Gendron, sociologue et Harvey Mead, fondateur de l'Union québécoise pour la conservation de la nature (Le Devoir), etc.

29 Les acteurs politiques sont particulièrement représentés dans les 163 articles analysés. Près de la moitié de ces articles font d'ailleurs de l'enjeu politique du DD une question centrale. 
dans la représentation du DD mise en avant par cette stratégie politique (acception rétrécie) et se distancie ainsi de sa propre représentation qui, comme nous l'avons vu, place les trois piliers du DD sur un quasi pied d'égalité, en donnant tout de même la primauté à l'économie et à l'environnement (acception large). En nous fondant sur Jégou (2007), nous considérons qu'il est légitime de penser que la couverture montréalaise du DD tend progressivement, elle aussi, vers une acception rétrécie du DD, à l'image de celle soutenue par le PPSDDCM et, plus généralement, par nombre des politiques durables actuelles (Richardson et Gordon, 2001 ; Cluzet, 2010). Cette acception est peutêtre plus facilement médiatisable, parce que plus sensationnaliste (thème hautement anxiogène des changements climatiques) et moins technique que l'acception médiane très théorique des années 1990, qui plaçait le DD à la jonction des piliers économie, environnement et société, mais qui semblait constamment relancer la question suivante: comment arriver réellement à un développement durable? Autrement dit, comment passer des mots aux actes, du discours à la pratique?

\section{Conclusion}

Cette étude a permis de vérifier partiellement nos deux hypothèses de recherche qui, rappelons-le, associent niveaux d'attention médiatique et action politique dans l'émergence d'une culture urbaine du développement durable à Montréal. Revenons sur quelques-uns des points saillants de notre analyse.

Tout d'abord, nous avons pu constater la reconnaissance médiatique accordée au thème du DD par les journaux La Presse, The Gazette et Le Devoir entre 1987 et 2009. Cette période peut être découpée en cinq temps forts d'attention médiatique: 1) introduction 1987-1992, 2) installation et évolutions timides 1993-1999, 3) essor 2000-2004, 4) apogée 2005-2007, et 5) relâchement relatif 2008-2009.

Ensuite, comme Lewis (2000), ou comme Holt et Barkemeyer (2010), nous avons pu mettre en évidence le caractère cyclique de l'attention des trois journaux analysés et constaté l'effet structurant d'un ensemble d'événements sur elle. D’après nos résultats, la mise en œuvre du Premier plan stratégique de développement durable de la collectivité montréalaise (2005-2009) est un événement déclencheur d’attention. En plus d'avoir officiellement placé le DD sur la scène montréalaise en établissant un nouveau standard en matière de politiques publiques urbaines et de considérations pour l'avenir de la cité, nos résultats montrent que ce plan s'inscrit dans l'apogée médiatique du thème du DD observée dans nos trois quotidiens au cours des années 2005, 2006 et 2007. Le relâchement de l'attention de la presse observé en 2008 et 2009 est également cohérent par rapport à la théorie des cycles d'attention médiatique de Downs (1972), qui associe l'intérêt politique engagé par la prise en charge institutionnelle d'un enjeu de société et le désintérêt médiatique pour ce même problème.

Cette remarque nous invite à considérer le futur de la médiatisation et de l'institutionnalisation du développement durable à l'échelle de Montréal. Signe de la progression sociale du discours du DD, à partir de 2005, même lorsque l'attention de la presse montréalaise se relâche, elle reste bien supérieure (en nombre d'articles) aux niveaux d'attention enregistrés durant la période 1987-2005, soit avant le PPSDDCM. Malgré le relâchement de l'attention en 2008, nous pensons que le thème du DD a peu de chance de lasser un jour la presse montréalaise, tant 
il est sans cesse renouvelé dans son acception et dans ses pratiques. L'analyse de contenu a d'ailleurs révélé que la presse montréalaise fait aujourd'hui preuve d'une plus grande originalité dans sa manière de couvrir la thématique du DD. Il est aisé de penser que cette tendance reflète aussi la diversité des secteurs de la société qui intègrent aujourd'hui les principes du DD, ne serait-ce que partiellement.

Au niveau de la Ville de Montréal, il semble que le DD soit durablement inscrit à l'agenda politique, comme en témoigne l'entrée en vigueur des deux autres plans stratégiques ayant succédé au PPSDDCM pour les périodes 2010-2015 et 2016-2020.

Parce qu'une recherche sérieuse ne saurait faire l'économie de sa propre critique, nous n'oublions pas que la définition des trois piliers du DD sur laquelle repose notre analyse comparative de contenu fait l'objet de nombreuses critiques, notamment pour son manque de lisibilité et l'opérationnalité qu'elle semble négliger (Mancebo, 2010). Toutefois, l'utilisation méthodologique de cette définition se justifie dans la mesure où c'est précisément cette définition qui est reprise - et souvent mal maîtrisée - par l'essentiel des acteurs. De prime abord, la métaphore des trois piliers peut paraître un peu simpliste. Néanmoins, cette simplicité apparente facilite grandement l'analyse de contenu, dans la mesure où chacun des trois piliers du DD renvoie à des champs thématiques bien précis et clairement définis dans la littérature scientifique. D'autres recherches pourraient inclure la notion de gouvernance, tant il est clair que c'est sa bonne gestion qui permet à toute démarche durable de s'installer, dans l'espace et dans le temps.

L'analyse comparative de contenus à laquelle nous avons eu recours a permis de dégager des grandes tendances qu'une analyse de discours permettrait certainement d'approfondir, sans contredire. De plus, il serait aussi intéressant de comparer la couverture de presse du DD à un éventail de sources officielles plus large que le seul Premier plan stratégique de développement durable de la collectivité montréalaise. Dans cette perspective, l'analyse de la Loi sur le développement durable, de 2006, (échelle du Québec) permettrait certainement de mettre en perspective nos résultats.

Les questions de recherche posées dans cet article entrevoient la dynamique suivante: la presse montréalaise favorise la conscientisation des responsables politiques, et les actions politiques subséquentes que ces derniers engagent viennent renforcer la médiatisation du DD. Nos résultats montrent que la presse montréalaise et les autorités publiques de Montréal s’influencent mutuellement, dans le temps et dans le sens qu'elles donnent au développement durable. Nous pensons néanmoins qu'elles sont aussi influencées par la progression du discours du développement durable à l'échelle globale.

À cet effet, Chomsky et Herman (2008) considèrent l'existence d'un filtre médiatique s'inscrivant dans le contexte étasunien. Intitulé «religion nationale et mécanisme de contrôle», ce filtre évolue selon le contexte géopolitique mondial des époques (l'anticommunisme d'après-guerre, la guerre contre le terrorisme, etc.). Les nombreux enjeux du développement durable font indéniablement partie du contexte particulier à notre époque. Dans cette perspective, nous pensons que l'urgence d'agir et le caractère particulièrement universaliste du développement durable expliquent, plus qu'on aurait pu l'imaginer, sa résonnance sur les scènes médiatique et politique montréalaises. 
La progression manifeste de l'attention des médias et de l'action politique en faveur du développement durable à Montréal nous confortent dans l'idée que la ville a bel et bien amorcé sa «Sustainability Revolution» (Edwards, 2005 : 21). Mais le développement durable inspire autant qu'il inquiète: les constats économiques, environnementaux (changements climatiques en tête) et sociaux sur lesquels il s'appuie laissent entrevoir un changement de paradigme que beaucoup souhaitent, mais que d'autres redoutent, voire freinent (Valenti, 2000; Brunel, 2008). On remarquera ainsi que l'idée du DD repose sur des ingrédients dont le succès est éprouvé et qui sont communs à la sphère médiatique autant qu'à la sphère politique: la peur et l'espoir. Selon Hamman (2012), le développement durable est l'occasion pour les élus, de reprendre l'initiative dans le domaine des politiques urbaines. Sans aller trop loin, on peut se demander si le discours alarmiste des médias ne donne pas également l'occasion aux acteurs politiques de se positionner en grands sauveurs de l'intérêt général et de renforcer ainsi leur légitimité démocratique auprès des citoyens. Aujourd'hui, l'intégration de considérations environnementales jusque dans les discours des politiques les plus radicales tend à laisser entendre cela.

Dans la suite de cette recherche, d'autres études pourraient envisager l'impact des différents biais médiatiques sur le discours des médias de masse en matière de développement durable, un concept qui entend revenir sur un modèle économique auquel les médias participent pourtant très largement. Il serait aussi intéressant d'étudier la manière dont le développement durable est aujourd'hui véhiculé et partagé par/dans les médias et les réseaux sociaux. En effet, une analyse de ces nouveaux espaces démocratiques permettrait d'étudier de manière plus directe le lien qui existe entre les représentations citoyennes et politiques du développement durable, et ce, sans passer par le prisme des médias. 


\section{Bibliographie}

AGENCE FRANCE PRESSE (2002) Qu'est-ceque le développement durable? La Presse, Montréal, 16 août 2002.

ANTON, Bernard (2009) Plaidoyer pour la terreet les vivants: essai sur l'éveil environnemental. Saint-Sauveur, Marcel Broquet.

BADDACHE, Farid (2006) Le développement durable au quotidien. Paris, Eyrolles.

BALTHAZARD, Bernard Louis (2006) Le développement durable face à la puissance publique. Paris, L'Harmattan.

BESANCENOT, François (2009) Territoire et développement durable: diagnostic. Paris, L'Harmattan.

BLAIN, Jean-François (2008) Développement durable: l'impasse économique. Le Devoir, Montréal, 28 février 2008.

BOURQUE, Jean-Louis (1996) Le développement durable vise-t-il à sauver la "plantète des riches »? La Presse, Montréal, 18 juin 1993.

BOUVIER, Véronique (2006) Le développement durable payant. La Presse, Montréal, 19 décembre 2006.

BOYKOFF, Maxwell T. et ROBERTS, J. Timmons (2007) Media coverage of climate change: current trends, strengths, weaknesses. Human Development Report 2007/2008 - Fighting climate change: human solidarity in a divided world, United Nations Development Programme.

BREGMAN, Dorine (1989) La fonction d'agenda: une problématique d'avenir. Hermès, nº 4 , p. 191-202.

BRUNE, François (1996) Les médias pensent comme moi! Fragments du discours anonyme. Paris, L'Harmattan.

BRUNEL, Sylvie (2008) À qui profite le développement durable? Paris, Larousse.

CALAMAI, Peter (1994) Sustainable development sounds a bit like diet ice cream. The Gazette, Montréal, 2 mai 1994.
CEM (CENTRE D'ÉTUDE SUR LES MÉDIAS) (2015) Portrait de la presse quotidienne [En ligne]. http://www.cem.ulaval.ca/ pdf/pressequotidienne.pdf

CHOMSKY, Noam et HERMAN, Edward S. (2008) La fabrication du consentement: de la propagande médiatique en démocratie. Marseille, Éditions Agone.

CLUZET, Alain (2010) Ville libérale ou ville durable? Répondre à l'urgence environnementale. Paris, L'Harmattan.

CMED (COMMISSION MONDIALE SUR L'ENVIRONNEMENT ET LE DÉVELOPPEMENT) (1989) Rapport Brundtland - Notre avenir à tous. Montréal, Éditions du Fleuve et les Publications du Québec.

CRE (CONSEIL RÉGIONAL DE L'ENVIRONNEMENT) (2010) Montréal Métropole du 21e siècle: plan quinquennal de développement 2010-2015 [En ligne]. http://credemontreal.qc.ca/wp-content/ uploads/2011/11/plan-quinquennal.pdf

DE PERTHUIS, Christian (2003) La génération future a-t-elle un avenir? Développement durable et mondialisation. Paris, Belin.

DIRECTION DE L'ENVIRONNEMENT ET DU DÉVELOPPEMENT DURABLE DE LA VILLE DE MONTRÉAL (2010) Premier plan stratégique de développement durable de la collectivité montréalaise: bilan synthèse 2005-2009 [En ligne]. https:// ville.montreal.qc.ca/pls/portal/docs/ page/d_durable_fr/media/documents/ bilan_2005-2009_plan_strat_dev_ durable_synth.pdf

DOWNS, Anthony (1972) Up and down with ecology: the "Issue Attention Cycle". Public Interest, vol. 28, p. 38-50.

EDWARDS, Andres R. (2005) The sustainability revolution: portrait of a paradigm shift. Gabriola, New Society Publishers.

EMELIANOFF, Cyria et STEGASSY, Ruth (2010) Les pionniers de la ville durable: récits d'acteurs, portraits de villes en Europe. Paris, Éditions Autrement. 
FELLI, Romain (2008) Les deux âmes de l'écologie: une critique du développement durable. Paris, L'Harmattan.

FLIPO, Fabrice (2007) Le développement durable. Rosny, Éditions Bréal.

FRANCOEUR, Louis-Gilles (1996) Retombée de l'Écosommet: Québec créera une organisation du développement durable. Le Devoir, Montréal, 28 juin 1996.

FRANCOEUR, Louis-Gilles (2002) Développement durable: le temps d'agir. Le Devoir, Montréal, 31 août 2002.

FRANCOEUR, Louis-Gilles (2006) Greenpeace: Charest travestit l'idée de développement durable. Le Devoir, Montréal, 15 mars 2006.

FRANCOEUR, Louis-Gilles (2007) Le développement durable, une idée souvent déformée. Le Devoir, Montréal, 30 mai 2007.

GAMMON, Mike (1998) Health issue is one of sustainability. The Gazette, Montréal, 27 juillet 1998.

GUMUCHIAN, Hervé, MAROIS, Claude et FÈVRE, Véronique (2000) Initiation à la recherche en géographie: aménagement, développement territorial, environnement. Montréal, Presses de l'Université de Montréal.

HAMMAN, Philippe (2012) Sociologie urbaine et développement durable. Bruxelles, De Boeck Supérieur.

HANSEN, Anders (1991) The media and the social construction of the environment. Media, Culture and Society, vol. 13, n ${ }^{\circ} 4$, p. 443-458.

HOLT, Diane et BARKEMEYER, Ralf (2010) Media coverage of sustainable development issues - attention cycles or punctuated equilibrium? Sustainable Development, vol.20, nº 1, p. 1-17.

HOWLETT, Michael (1997) Issue-attention and punctuated equilibria models reconsidered: an empirical examination of the dynamics of agenda-setting in Canada. Canadian Journal of Political Science, vol. 30, nº 1, p. 3-29.

JÉGOU, Agnès (2007) Les géographes français face au développement durable. L'Information géographique, vol.71, p. 6-18.
JUILLET, Luc et ANDREW, Caroline (1999) Développement durable et nouveaux modes de gouvernance locale: le cas de la Ville d'Ottawa. Économie et Solidarités, vol. 30, n² 2, p. 75-93.

KEATING, Michael (1993) Covering the environment: a handbookon environmental journalism (National Round Table on the Environment and the Economy). Ottawa, The Graduate School of Journalism, The University of Western Ontario.

LAFRANCE, Gaëtan et LAFRANCE, Julie (2014) Qui peut sauver la cité? Montréal, Éditions Multimondes.

LEAL FILHO, Walter (dir.) (2000) Communicating Sustainability. Amsterdam, Peter Lang Scientific.

LEAL FILHO, Walter et BORNER, Joachim (2005) Communicating sustainable development: a practical guide for communications and media professionals - Part 2: mass media and sustainability [En ligne]. http://www. wbcsd.org/web/complus/documents/ communicating_sd_chapter2.pdf

LERAY, Christian (2008) L'analyse de contenu: de la théorie à la pratique - La méthode Morin-Chartier. Québec, Presses de l'Université du Québec.

LEWIS, Tammy (2000) Media representations of 'Sustainable Development': sustaining the Satus Quo? Science Communication, vol. 21, p. 244-273.

MANCEBO, François (2010) Développement durable. Paris, Armand Colin.

McCOMBS, Maxwell, EINSIEDEL, Edna et WEAVER, David (1991) Contemporary public opinion: issues and the news. New Jersey, Lawrence Erlbaum Associates.

McKIBBEN, Bill (2008) American earth: environmental writing since Thoreau. New York, Literary Classics of the United States.

MÉTROPOLIS (ASSOCIATION MONDIALE DES GRANDES MÉTROPOLES) (2015) IVe Congrès Mondial METROPOLIS à Montréal, "Le citoyen et le développement durable» [En ligne]. http://www. metropolis.org/fr/timeline/ive-congresmondial-metropolis-montreal-le-citoyenet-le-developpement-durable 
MOREAU DEFARGES, Philippe (2008) La mondialisation. Paris, Presses Universitaires de France.

MUCCHIELLI, Roger (2006) L'analyse de contenu: des documents et des communications. Issy-les-Moulineaux, ESF éditeur.

NITZ, Michael (2000) The media as a tool for communication on the envrionment and sustainability. Dans Walter Leal Filho (dir.) Communicating Sustainability. Amsterdam, Peter Lang Scientific, p. 45-67.

NOLLET, Jérémie (2009) ST 14: L'analyse des politiques publiques existe-t-elle encore? Session 3: Politiques publiques et regards croisés: frontières et disciplines mixtes Croiser analyse des politiques publiques et sociologie des médias: genèses et usages des concepts de miseà l'agenda et de construction des problèmes. $10^{\mathrm{e}}$ Congrès de l'association française de science politique, Grenoble, 7-9 septembre 2009 [En ligne]. http://www. congresafsp2009.fr/sectionsthematiques/ st14/st14nollet.pdf

ONU (ORGANISATION DES NATIONS UNIES) (2000) Éliminer la pauvreté, c'est possible: objectifs du millénaire pour le développement et l'après-2015 [En ligne]. http://www.un.org/fr/millenniumgoals/

PADIS, Marc-Olivier (2009) Après le krach et avant Copenhague: de quelle "reprise» parle-t-on? Esprit, vol. 12, n³60, p. 51-58.

RABOY, Marc (2000) Les médias québécois: presse, radio, télévision, inforoute. Boucherville, Gaëtan Morin Éditeur.

RICHARDSON, Harry et GORDON, Peter (2001) Land management for sustainable urban regions workshop: sustainable Portland? A critique, and the Los Angeles counterpoint. Conference of the Association of Collegiate Schools of Planning, Cleveland, 10 novembre 2001.

RIOUX SOUCY, Louise-Maude (2005) Lepoint de vue féminin sur le développement durable. Le Devoir, Montréal, 21 janvier 2005.
SACHSMAN, David (2000) The role of the mass media in shaping perceptions and awareness of environmental issues. Climate Change Communication Conference, Waterloo, 22-24 juin 2000.

STATISTIQUE CANADA (2011) Données du Programme de Recensement de la Population de 2011 [En ligne]. http:// www12.statcan.ca/census-recensement/ index-fra.cfm

SUSTAINABILITY (2002) Good news and bad: the media, corporate social responsability and sustainable development. London, Ketchum and United Nations Environment Program.

THEYS, Jacques (2002) L'approche territoriale du «développement durable», condition d'une prise en compte de sa dimension sociale. Développement durable et territoires [En ligne]. http://developpementdurable. revues.org/index1475.html

TRUDELLE, Catherine (2005) Visibilité de la participation des femmes aux conflits urbains à Québec, 1965-2000. Québec, Université Laval, École supérieure d'aménagement et de développement, thèse de doctorat.

VALENTI, JoAnn Myer (2000) A review of the President's Council on sustainable development (U.S.): building networks, throwing pebbles at a Goliath media. Dans Walter Leal Filho (dir.) Communicating sustainability. Amsterdam, Peter Lang Scientific, p. 121-133.

VIVIEN, Franck-Dominique (2003) Jalons pour une histoire de la notion de développement durable. Mondes en développement, $\mathrm{n}^{\circ} 121$, p. 1-21.

WACKERMANN, Gabriel (2000) Géographie urbaine. Paris, Ellipses-Marketing.

YANOVITSKY, Itzhak (2002) Effects of news coverage on policy attention and actions: a closer look into the media-policy connection. Communication Research, vol. 29, nº 4 , p. 422-451. 